\title{
COMPLETE DISINFECTION OF ROOT CANALS
}

Sirona SiroLaser from Sident Dental Systems can enhance endodontic results by helping ensure complete disinfection of root canals, thus eliminating at least one source of reinfection and consequent endodontic failure.

SiroLaser can also be used for soft tissue surgery in a variety of cosmetic dentistry applications as well as crown lengthening, treating periodontal

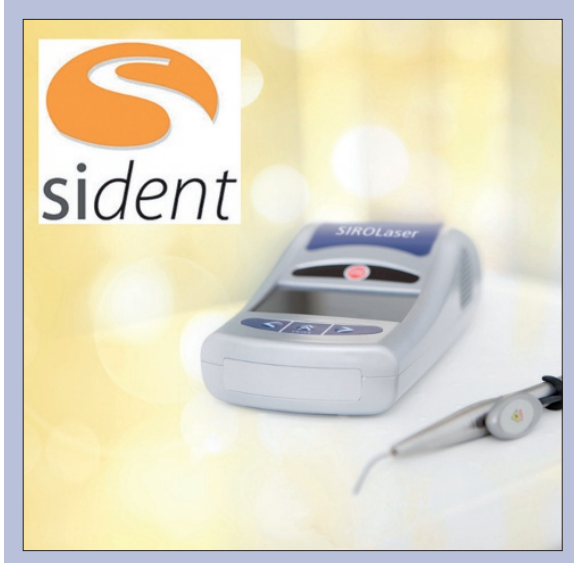

pockets, resolving peri-implantitis, and bleaching applications.

In surgical applications it offers high precision tissue removal with minimal trauma to surrounding tissues, minimised bleeding for clearer visibility, protection against post-operative infection, minimised scar formation, an enhanced healing response and virtually no postoperative pain.

Weighing $450 \mathrm{~g}$, it features the very latest diode technology which means that the unit size is kept to a minimum. The control unit has a clear menu of easy to select functions, while its LED display shows all the treatment parameters.

The ergonomically designed, fully autoclavable handpiece can be operated by either finger switch or foot control and can be easily integrated into the treatment workflow. Reader response number 63

\section{HANDS-ON COURSE}

Henry Schein Minerva's 'Twisted file: innovation in endodontics' course is a hands-on course designed for general practitioners to improve their daily endodontic skills.

Areas covered will include diagnosis, case selection, the latest endodontic instrumentation, and the cleansing, shaping and packing of the root canal system in three dimensions with warm obturation techniques and bonded obturation.

On completion, delegates should have a comprehensive understanding of the key elements of shaping root canals, how rotary NiTi files can improve root canal treatments for the GDP, the importance of cleaning the root canal system in the preparation for shaping and filling, and the importance of a three dimensional fill.

The course qualifies delegates for three hours of verifiable CPD and will take place on 19 March in Wakefield and 25 March in Glasgow. Email education@henryschein.co.uk for more information.

Reader response number 64

\section{CREATE BALANCE AND MOBILITY}

Bambach's unique saddle seat design allows the spine and pelvis to work together to create balance and mobility in the upright position.

As a result good posture happens naturally as you sit on stool, which encourages 'dynamic' seating. Due to the unique design of this stool, your feet are free to move to and from your foot pedal without altering your pos- ture, further promoting health. This helps not only to reproduce the stress free ' $S$ ' shape of the spine but also to maintain it effortlessly all day long.

Bambach stools are available in a wide variety of colours and finishes to harmonise with the modern dental practice and there is also a free 30 day trial period.

Reader response number 65 